\title{
One too many: intellectual disability secondary to undiagnosed phenylketonuria
}

Joannie Hui ${ }^{1}$ *, FRCP (Edin), FRACP, SC Chong1', FHKCPaed, FHKAM (Paediatrics), LK Law², PhD, FRCPath, LK Lee ${ }^{1}$, FHKCPaed, MPH (HK), Sandy Chang ${ }^{3}$, RD, BSc (Hons) Nutrition/Dietetics, Phyllis Yau ${ }^{3}$, RD, PgDip Dietetics, YP Yuen², FHKCPath, FHKAM (Pathology)

Departments of ${ }^{1}$ Paediatrics and ${ }^{2}$ Chemical Pathology, The Chinese University of Hong Kong, Shatin, Hong Kong;

${ }^{3}$ Dietetics Department, Prince of Wales Hospital, Shatin, Hong Kong

Hong Kong Med J 2016;22:506-8

*Corresponding author: joanniehui@cuhk.edu.hk

\section{Case report}

Hyperphenylalaninaemia refers to the clinical condition characterised by increased amounts of phenylalanine in blood and other tissues. It can result from either a deficiency of phenylalanine hydroxylase $(\mathrm{PAH})$ or defects in synthesis or regeneration of tetrahydrobiopterin (BH4), a cofactor for PAH (Fig $1)$.

We describe a 2-year-old boy who was referred by the Maternity Child Health Clinic to the Department of Paediatrics in June 2014 for assessment of developmental delay. He was the second child in his family, born at term and following an uneventful pregnancy to non-consanguineous Chinese parents. His birth weight was $3475 \mathrm{~g}$. He was formula-fed from birth, then gradually weaned to a normal toddler diet. Family history was unremarkable. The mother was from Hubei, a province in Central China, and father was from Shenzhen, a major city in Southern China. The 5-year-old brother was well and there were no developmental concerns.

The boy's early developmental milestones were unremarkable. He developed social smile at 1 month of age, sat unsupported at 8 months, and walked independently at 1 year and 8 months. He started saying single words at 15 months but at the age of

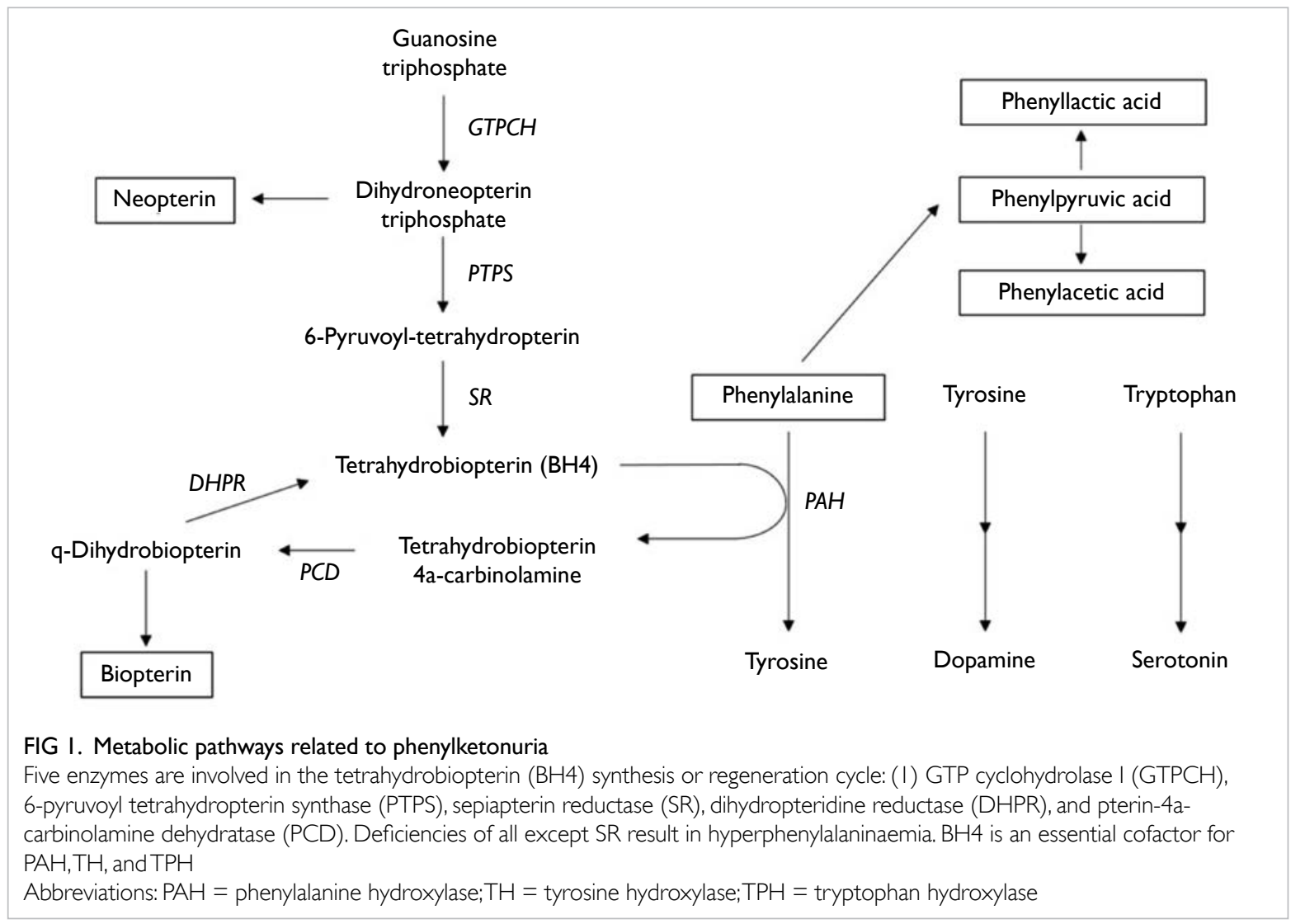


2 years was still saying only a few single words and no phrases. Also he was noted to be hyperactive with behaviour that was at times difficult to control. His physical growth was satisfactory. His head circumference was $48.5 \mathrm{~cm}$ (50th centile), weight $14.1 \mathrm{~kg}$ (90th centile), and height $87 \mathrm{~cm}$ (50th centile). There were no dysmorphic features and no abnormalities were detected on physical examination. His hair was slightly brownish.

Initial baseline investigations revealed normal blood count and liver, renal, and thyroid function. Urine organic acid analysis showed markedly elevated phenylalanine metabolites including phenyllactic, 3-phenylpyruvic, and phenylacetic acids. Plasma phenylalanine level was markedly elevated at $1948 \mu \mathrm{mol} / \mathrm{L}$ (reference range [RR], 26-91 $\mu \mathrm{mol} / \mathrm{L}$ ) and tyrosine was $47 \mu \mathrm{mol} / \mathrm{L}$ (RR, 24-115 $\mu \mathrm{mol} / \mathrm{L})$. Urine biopterin was mildly elevated at 5.0 $\mu \mathrm{mol} / \mathrm{mmol}$ creatinine (RR, 0.5-3.0) while neopterin was normal at $1.7 \mu \mathrm{mol} / \mathrm{mmol}$ creatinine (RR, 1.14.0). Erythrocyte dihydropteridine reductase activity was normal. A BH4 loading test was performed to check for BH4 responsiveness according to standard protocol. ${ }^{1}$ Blood for phenylalanine and tyrosine was checked serially at 8,16 , and 24 hours after each administration of $20 \mathrm{mg} / \mathrm{kg} \mathrm{BH} 4$ (Kuvan) orally on day 1 and day 2 of the loading test. No appreciable drop in blood phenylalanine level was observed confirming $\mathrm{BH} 4$ non-responsiveness.

All coding exons and flanking introns of the $P A H$ gene (reference sequence NM_000277.1) were sequenced using the standard Sanger method. A heterozygous missense mutation c.860T $>C$ was detected in exon 8 of the $P A H$ gene. This mutation changes the highly conserved leucine at position 287 to proline (p.Leu287Pro) [Fig 2]. Another mutation affecting the same amino acid p.Leu287Gly has been reported previously in patients with phenylketonuria (PKU). ${ }^{2}$ In-silico analyses by four prediction software (PolyPhen-2, SIFT, Mutation Taster, PON-P2) also consistently predicted that the mutation is pathogenic. Therefore, $P A H$ c.860T $>C$ (p.Leu287Pro) is highly likely to be a pathogenic mutation. The mother was a carrier of this missense mutation. $P A H$ gene dosage analysis by multiplex ligation probe amplification (SALSA MLPA probemix P055-C1 PAH) did not detect any $P A H$ gross deletion or duplication. Therefore, the second PAH mutation of this patient remained unidentified.

The patient was started on a phenylalaninerestricted diet after diagnosis. Special formula XP-2 powder from SHS was used as the phenylalaninefree and tyrosine supplement source. With dietary advice and close supervision, his phenylalanine levels gradually came down to 300 to $500 \mu \mathrm{mol} / \mathrm{L}$ while tyrosine levels were maintained at 50 to 80 $\mu \mathrm{mol} / \mathrm{L}$ over the following 6 months. It is too early to report on his progress in terms of development and behaviour consequent to better control of the blood phenylalanine level.

\section{Discussion}

We believe this is the first reported case of a locally born child with classic PKU in Hong Kong. Although a low phenylalanine diet was commenced promptly upon diagnosis, the cognitive impairment that has occurred as a result of the unrecognised long-standing hyperphenylalaninaemia is likely irreversible. Other than this patient reported here, two other classic PKU patients are being followed up at the authors' metabolic clinic. Both patients were born in Guangzhou, the capital city of Guangdong province in South China. They were diagnosed through the newborn screening programme in Guangdong province. With good dietary compliance, both children have achieved normal growth and development.

This year marked the 56th anniversary of PKU newborn screening. Effective newborn screening programmes worldwide including those in China have identified thousands of infants with PKU and prevented intellectual disability through early diagnosis and treatment. Yet in Hong Kong the need for PKU screening has never been seriously addressed. Over the last 30 years, newborn screening in Hong Kong has remained unchanged with cord blood screening for G6PD (glucose-6-phosphate dehydrogenase) deficiency and congenital hypothyroidism. The current practice of newborn screening lags behind the rest of the world, and has never been challenged because for

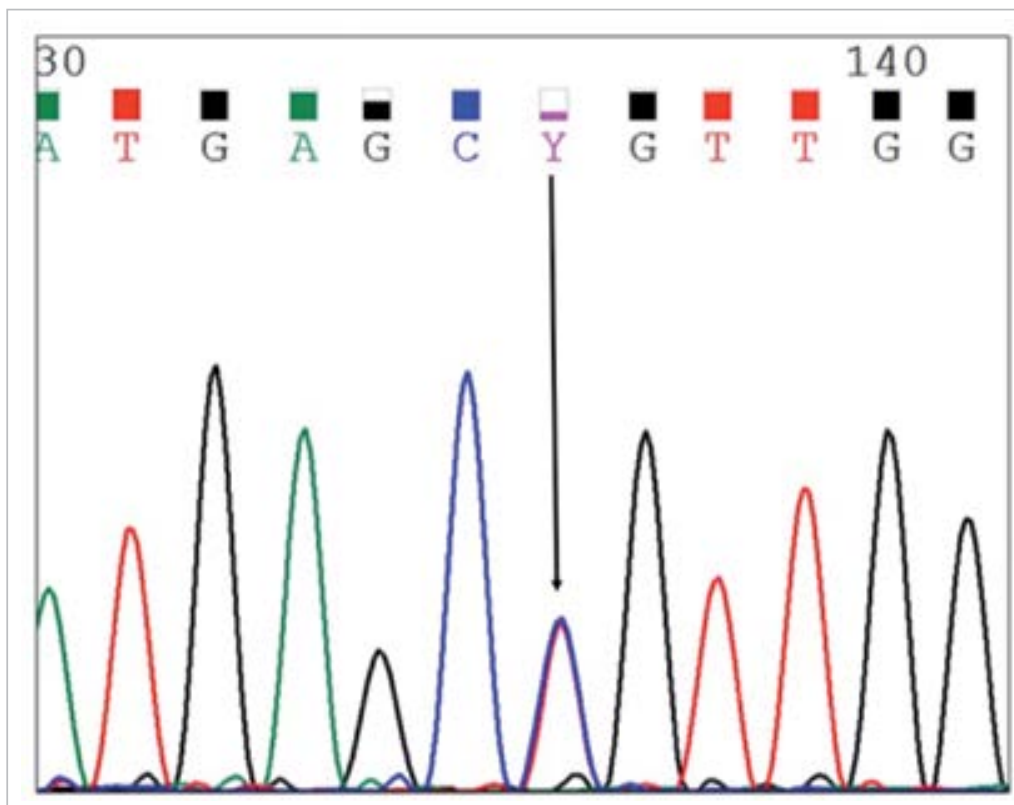

FIG 2. Electropherogram of the PAH mutation NM_000277.I:c.860T>C (p.Leu287Pro)

The arrow indicates the position of mutation 
years there have been only scant cases of 6-pyruvoyl tetrahydropterin synthase (PTPS) deficiency and no classic PKU cases reported locally. In the absence of newborn screening, why has only PTPS deficiency and no classic PKU patients been identified in Hong Kong? One reason could be that individuals with PTPS deficiency often present with more complex neurological manifestations and are more likely to undergo extensive investigations. On the contrary, those with classic PKU present with variable degrees of intellectual disability and behavioural problems and no overt neurological signs, and may not have been as extensively investigated. In addition, there is a general misconception among practising clinicians in Hong Kong that PKU is a disease of the Caucasian population. Even if it does affect the Chinese population, only the Northern Chinese are affected. As such, plasma amino acid or urine organic acid profile may not have been routinely requested for investigation in children with unexplained developmental delay, intellectual disability, behavioural problems, or autistic spectrum disorders. Further, these investigations may not be as widely available in non-hospital-based private laboratories.

Without a territory-wide newborn screening programme, it is impossible to ascertain the true incidence of PKU in Hong Kong. Irrespective of whether Hong Kong does have a different PKU incidence compared with the rest of China, the presence of confirmed cases locally provides a strong argument for diagnosis and treatment of affected individuals at the earliest instance rather than after symptomatic presentation. Hong Kong cannot afford to have more intellectual disability as a result of the unavailability of PKUscreening. Until this programme becomes universally available, we advocate plasma amino acid and urine organic acid analysis to be incorporated into the diagnostic workup for all children with unexplained developmental delay, intellectual disability, behavioural problems, and autistic spectrum disorders.

\section{References}

1. Blau N, Hennermann JB, Langenbeck U, Lichter-Konecki U. Diagnosis, classification, and genetics of phenylketonuria and tetrahydrobiopterin (BH4) deficiencies. Mol Genet Metab 2011;104 Suppl:S2-9.

2. Bardelli T, Donati MA, Gasperini S, et al. Two novel genetic lesions and a common BH4-responsive mutation of the $P A H$ gene in Italian patients with hyperphenylalaninemia. Mol Genet Metab 2002;77:260-6. 